\title{
Intergenerational differences in acculturation experiences, food beliefs and perceived health risks among refugees from the Horn of Africa in Melbourne, Australia
}

\author{
Alyce Wilson ${ }^{1}$ and Andre Renzaho ${ }^{2,3, *}$ \\ ${ }^{1}$ School of Medicine, University of Melbourne, Melbourne, Victoria, Australia: ${ }^{2}$ Migration, Social Disadvantage, \\ and Health Programs, International Public Health Unit, School of Public Health and Preventive Medicine, Monash \\ University, Level 3, Burnet Building, 89 Commercial Road, Melbourne, VIC 3004, Australia: ${ }^{3}$ Centre for \\ International Health, Burnet Institute, Australia
}

Submitted 22 March 2013: Final revision received 17 0ctober 2013: Accepted 24 0ctober 2013: First published online 14 January 2014

\begin{abstract}
Objective: To investigate the differences in acculturation experiences between parent and adolescent refugees from the Horn of Africa in Melbourne, Australia and to explore food beliefs and perceived health risks from an intergenerational perspective.

Design: Qualitative cross-sectional study involving a combination of semi-structured one-on-one interviews and focus group discussions.

Setting: North-West suburbs of Melbourne, Australia.

Subjects: Eritrean, Ethiopian, Somali and Sudanese refugees.

Results: Using a purposeful sampling technique, twelve semi-structured face-to-face interviews (nine adults and three adolescents) and four in-depth focus groups (two with adolescents each containing six participants and two with adults one containing six participants and the other ten participants) were carried out. Thus overall data were obtained on fifteen adolescents and twenty-five parents. Qualitative analysis identified differences between parents and adolescents in relation to lifestyle, diet and physical activity. Views regarding health consequences of their changed diets also differed. Parental feeding practices encompassed a variety of methods and were enforced in an attempt by parents to control their children's dietary behaviours and prevent their drift away from traditional eating habits. Conclusions: These findings call for more research to contextualise dietary acculturation among refugee youth and the impact of migration on parenting styles and feeding practices in communities from the Horn of Africa. Preventive health programmes with Horn of Africa refugees need to acknowledge the effect of acculturation on diet and physical activity levels and a socio-cultural framework needs to be developed with respect to the importance and influence of the family environment.
\end{abstract}

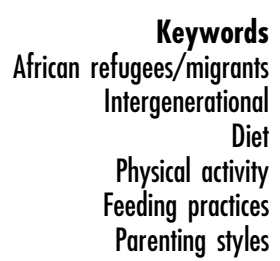

Both natural and human-made disasters as well as poverty have resulted in increased population displacements. The UN Department of Economic and Social Affairs estimates that in 2011 there were 214 million international migrants worldwide, representing $3 \cdot 1 \%$ of the world's population, which does not include the 33.9 million people who were refugees, asylum seekers, internally displaced people and stateless persons ${ }^{(1)}$. Extensive numbers migrate from lowand middle-income countries to high-income countries, either as part of voluntary migration (e.g. economic/skilled migration or family reunion) or forced migration (e.g. refugees and asylum seekers) ${ }^{(2)}$. With the 2011 census revealing that $26 \%$ of the Australian population were born overseas $^{(3)}$, Australia remains a preferred destination for both voluntary and forced migrants. A significant number of sub-Saharan African migrants have relocated to Australia under the Refugee and Humanitarian Entrants Scheme and now represent $1 \%$ of the Australian population ${ }^{(3)}$.

Once in Australia, migrants from developing countries often experience health inequalities due to their generally poor socio-economic position relative to the average Australian. Migrants are more likely than their Australianborn counterparts to experience poverty (i.e. insufficient resources and income) and to be on low incomes, live in rental accommodation and to experience social exclusion due to loss of networks ${ }^{(4)}$. Hence, migration from sub-Saharan Africa to affluent, Westernised countries is associated with an increased risk of chronic disease, especially obesity and diabetes ${ }^{(5)}$. Data from Ghanaian migrants in Sydney ${ }^{(6)}$, Ethiopian migrants in Israel ${ }^{(7,8)}$ and 
African refugees in Australia ${ }^{(9,10)}$ and the USA ${ }^{(11)}$ indicate that African refugees and migrants to industrialised countries gain weight rapidly and are at increased risk of obesity-related chronic diseases following migration. The underlying mechanisms which predispose migrants relocating from developing to developed countries to an increased risk of obesity and obesity-related diseases are complex and multifaceted ${ }^{(12)}$. There is currently no framework which can be used to assist researchers and health professionals to understand the post-migration risk factors that predispose sub-Saharan African migrants to obesity and obesity-related diseases.

There is some emerging evidence that lifestyle changes and dietary acculturation are key factors ${ }^{(13)}$. The term 'acculturation' has been used in the literature to describe both the cultural and psychological changes that occur when two cultures come into contact with each other ${ }^{(14)}$. While a number of acculturation domains have been identified in the literature (see Flannery et al. ${ }^{(15)}$ and Padilla and Perez ${ }^{(16)}$ ), they fall under two models of acculturation: the linear acculturation model and the bi-dimensional acculturation model. The discussion of these models is beyond the score of the present paper. Nevertheless, lifestyle changes that sub-Saharan African migrants experience following migration to Australia are better explained by the bi-dimensional acculturation model, which describes four coping mechanisms associated with acculturation: (i) integration (accepting both the host culture and the culture of origin as a way of life); (ii) assimilation (rejecting the culture of origin and fully accepting the host culture as a way of life); (iii) marginalisation (rejecting both the host culture and the culture of origin as a way of life); and (iv) traditional (maintenance of the culture of origin and rejection of the host culture as a way of life $)^{(9)}$. These coping strategies dictate how individuals interact with their environment and access and utilise available health promotion programmes ${ }^{(17)}$.

Therefore, migrating to Australia presents sub-Saharan African migrants with a new food environment and the ways in which refugees and migrants understand and negotiate the different food system can impact their food choices, dietary habits and subsequent chronic disease risk $^{(18)}$. Poor health literacy offers an alternative explanation. This refers to the 'degree to which individuals have the capacity to obtain, process and understand basic health information and services needed to make appropriate health decisions, ${ }^{(19)}$. Available data indicate that a low level of health literacy is independently associated with poor nutrition and health. However, for migrant populations, young people often demonstrate adequate knowledge of nutritional and health risks due to better English proficiency than their parents, school attendance or following health programmes on television. Hence children can become a reliable source of health promotion at the family level. Whether this is true for Horn of Africa refugees and migrants, most of whom migrate to industrialised countries from refugee camps and with little English, is unknown. Therefore, the purpose of the present study was to examine the attitudes and beliefs about food and perceived health risks among Horn of Africa migrants settling in Melbourne, Australia.

\section{Methods}

\section{Design and participant recruitment}

An exploratory qualitative research design was employed to assist the researchers to understand the acculturation experiences, beliefs about food and perceived health risks of refugees from the Horn of Africa. A thorough account of the study design and participant recruitment has been previously described ${ }^{(18)}$. Briefly, participants were Eritrean, Ethiopian, Somali and Sudanese refugees living in the North-West suburbs of Melbourne, Australia. Using purposeful sampling, participants were recruited with the assistance of the African Review Panel ${ }^{(10)}$ and through the African Family Relationship Centre, the North Yarra Community Health Centre, the Centre for Multicultural Youth Issues and the Ethiopian Community Association. Through activities provided by these organisations such as women's art and craft classes, girl's groups, English classes, indoor soccer competitions and church choir, the researchers met with the community members and explained the study's objectives and its intended outcomes to them through a plain language statement. After the study was explained, participants were invited to take part and the researchers stressed that taking part was voluntary. Those who accepted to take part were asked to sign a consent form. A total of forty participants consented to take part in the study.

\section{Data collection}

A two-pronged approach was used to enhance data richness. In the first instance, twelve semi-structured face-to-face interviews were carried out (nine parents and three adolescents). After conducting the twelve interviews, key concepts which required further dialogue in focus groups were uniformly identified by adult and adolescent participants. Therefore, the major themes identified in the initial face-to-face interviews were further explored during focus group discussions. A total of four focus group discussions were carried out. Of these, two were with adolescents and each contained six participants $(n$ 12) and the other two were with parents (one containing six participants and the other ten participants, $n$ 16). A literature review ${ }^{(20)}$ informed the framework of the interview guides which were carefully reviewed and expanded upon in consultation with the African Review Panel. Interview guides were developed separately for adults and adolescents. Interviews and focus groups were conducted in familiar venues for 
participants including homes, community centres, churches and sporting centres. Data were collected until the researchers were satisfied that the data were rich enough and covered adequate dimensions of the topics being investigated (data saturation). All interviews and focus groups were conducted in English or the given community language with the aid of a professional interpreter.

To minimise threats to authenticity and validity of interpreted accounts arising from the translation by the interpreter from English to community languages, multiple strategies were utilised. First, all participants agreed to have their voices audio recorded except six, in which case detailed notes were taken and checked with participants at the end of the session. The tape and field notes were independently reviewed by the African Review Panel members (all of whom are bilingual) to establish the reliability of responses and their comments were taken into account when analysing the data. Second, participants were told that all interviews would be confidential and under no circumstances were interpreters to discuss the information imparted during the interview with any other person outside the research team. Finally, to maintain the interpreters' degree of independence, they were selected from outside the communities being interviewed.

For the semi-structured interviews, the first 10 min were spent getting the consent forms signed and collecting demographic data. Once demographic characteristics were obtained the interview lasted approximately 20-40 min depending on the level of English literacy. For the focus group discussions, consistent with the African cultures ${ }^{(18)}$, a platter of fruit and a light meal were provided at the beginning of the focus group discussion to allow participants to intermingle, sign consent forms and obtain demographic data. This process took about $20 \mathrm{~min}$. Then the participants answered questions for an additional $40 \mathrm{~min}$ to $1 \mathrm{~h}$. Interviews and focus group discussions were transcribed verbatim and participants were given the opportunity to check and verify discussions recorded and notes written.

\section{Data analysis}

Interview transcripts were manually analysed using a thematic analysis approach as this enabled the researchers to inductively find themes in the data and expose concepts that were not preconceived or anticipated ${ }^{(21)}$. Open coding was used by the researchers (A.W. and A.R.) to code responses from transcripts and field notes. A process of focused coding and constant comparison was used to consolidate the code list and re-sort concepts into groups until clear, consistent themes emerged. Emerging themes were discussed between the researchers until a consensus was reached. Key themes were then described and interpreted by the researchers and narrative responses selected to best illustrate the findings and themes.

\section{Results}

Forty Horn of Africa refugees took part in the study (Table 1). Twenty-five $(62 \cdot 5 \%)$ were adults and fifteen $(37 \cdot 5 \%)$ were adolescents. Of the forty participants taking part in the study, twelve took part in face-to-face semistructured interviews and twenty-eight in focus group discussions. Eighteen were Somali $(45 \cdot 0 \%)$, thirteen were Sudanese $(32 \cdot 5 \%)$, five were Ethiopians (12.5\%) and four were Eritreans (10.0\%). Participants' demographic profile is summarised in Table 1.

Three distinct themes emerged from the thematic analysis: (i) acculturation; (ii) food beliefs and perceived health consequences; and (iii) parental feeding practices. All themes emerged simultaneously from both the parents' and adolescents' data regardless of ethnicity (as defined by country of birth), except for parental feeding practices which was isolated to the parents' findings.

\section{Theme 1: Acculturation Parents' views}

Among parents, two main forms of acculturation were evident: changes in lifestyle and change in dietary practices (dietary acculturation).

\section{Lifestyle changes}

In terms of changes in lifestyle, parents spoke of a significant lifestyle change, from a traditional, easy-going, community-oriented lifestyle to one which was hectic and time-constrained. Changes in lifestyle were also described in relation to differences in the amount of physical activity and manual work undertaken in Africa compared with Australia. Parents described how they were able to maintain a healthy weight in Africa from walking as the main source of transport and partaking in significant amounts of manual labour. Since arriving in Australia they considered themselves to be more sedentary, having a greater reliance on cars for transport and spending long hours sitting at their desk at work and in front of the television at home. Parents also commented on the cooler climate in Melbourne and lack of recreational areas such as parks and open spaces. The majority of parent participants lived in high-rise estates and hence they felt limited in their capacity to exercise and play with their children. In regard to diet, parents described how their change in lifestyle had similarly changed their dietary habits. Working long hours and having a generally busy life often persuaded them to opt for food which was quick and easy. One Somali mother summed up the change in lifestyle, pervasively presented by participants:

'So it's a different lifestyle... you are in the office or you are in the house. Every day you start the same, tomorrow you coming work, 5 o'clock you finish, you go home. The only thing you can do is cook and clean inside the house. No time even to walk, 
no time to exercise... In Somalia I used to walk all day... You walk a lot [in Africa], it's a kind of healthy lifestyle there. So it's completely another world.' (Maca, 34 years)

An Eritrean mother added:

'People are very active [in Africa]. Not many people have cars. People move a lot. Walk a lot too. ... the weather is hot and sweaty. But here [in Australia] we use car for little distance. Sit at home in front of the TV. That's why people get diabetes... I haven't been going out. And the kids, no backyard to run. You see there is no yard... House is small, nowhere to move.' (Salome, 39 years)

\section{Dietary acculturation}

In terms of changes in dietary practices, dietary acculturation emerged as a significant indication of acculturation. Dietary changes occurred in many forms. The most recurring forms included children's preference for Australian food (Table 2), choosing Australian food over traditional food for convenience, changes to shopping and cooking habits, changes to meal formats, decreased family commensality and altered social roles with women entering the workforce. Parents described how children would choose Australian food to fit in with peers. In terms of meal format changes, parents described the biggest change was in relation to lunch which they believed was the most important meal of the day in Africa. Parents explained how families would come home from work and school to eat a hot, freshly cooked meal together, have a rest and then return to their daily activities for the rest of the afternoon. However, in Australia parents felt there was a greater emphasis on the evening meal as the main family meal. Many of the mothers had compensated for the loss of the shared family lunch by adopting a late afternoon/early evening lunch (4-5 pm) after their children had come home from school. This meal would be followed by a light supper later on in the evening consisting of cereal, toast or fruit. A Somali mother explained the importance of lunch:

'Lunchtime is most important... In Somalia, the main meal at lunchtime... That's when you have the family meal.' (Roberta, 46 years)

Another Somali mother felt that her family was losing its culture by not sharing the main meal with the immediate family in the middle of the day:

'...the lunch is not the way it used to be in Somalia because people [are] busy and kids have school and the lunchtime usually back home, we don't have that culture [in Australia] anymore you know. Lunchtime people come together and eat together, sometimes it is happening here but it is not the culture we used to have, its different now.' (Clarice, 34 years) 
Table 2 Australian foods eaten by adolescents as identified by parents and adolescents; refugees from the Horn of Africa in Melbourne, Australia

\begin{tabular}{ll}
\hline Food category & Food items \\
\hline Condiments & Nutella \\
& BBQ sauce \\
& Jam \\
& Peanut butter \\
& Cheese \\
Snack foods & Chips (packets) \\
& Muffins \\
& Yoghurt \\
& Two minute noodles \\
& Chicken nuggets \\
& Toast (sliced bread) \\
Jelly \\
Beverages & Pepsi \\
& Coca-Cola \\
Takeaway foods & Juice \\
& McDonalds \\
& KFC \\
& Pizza \\
& Chicken/beef burgers \\
& Kebabs ('Ali Baba') \\
Nandos \\
Fish and chips \\
Noodles \\
Lasagne \\
Hot dogs \\
Meat piest \\
Sandwiches \\
Pasta \\
\end{tabular}

*Predominantly Sudanese.

thalal pies for Muslims.

A Sudanese mother explained how she had adjusted the timing of the lunch meal to fit in with the different school times in Australia:

'...I cook for the lunchtime. The lunchtime is when the children come back straight away from school... Yeah 3.30 to 4 is when the mothers always stay home and cook and when the children come back they eat the main meal.' (Mohamudu, 30 years)

Interestingly, women described feelings of identity loss as their role as the family cook. Changed social roles manifested in response to changed meal formats and women spoke about how their husbands were taking on a greater role in food preparation and provision as they entered the workforce and worked long hours. Parents explained that in Africa every meal was prepared at home by the women to be eaten together as a family, yet in Australia this differed. First, the increased number of food choices available in Australia meant that children had more variety to choose from. Second, as children became more accustomed to the 'individualistic' society present in Australia they were empowered to make their own choices, including their own food choices, and did not feel compelled to eat the same food as the rest of the family. Mothers were distressed by this and felt that family commensality was deteriorating. In collective societies such as Africa, mothers often demonstrate and reaffirm their identity through cultural practices, such as preparing traditional family meals. In Australia, dietary acculturation appeared to have impacted on family commensality and hence the mothers' sense of identity and purpose. A Somali mother described how the breakfast meal had been disrupted since migrating to Australia:

'I miss back home our traditional breakfast, we used to have every day. We eat injera and breakfast is prepared for the whole family and everyone knows the time for the breakfast, everyone eats together... but that's not the way anymore here... one child wants weet-bix and one child wants slice toast and then you see seven people in my house and everyone wants [something different]... back home we used to have one breakfast meal, and everyone used to eat [the] same... it's really a lot of cultural food habits and all that we miss a lot. So that's the problem... [I] feel no more of that happiness anymore...' (Olga, 39 years)

This was further illustrated by an Ethiopian mother:

'It takes time to cook our food. You need time. And I'm working full time... I'm not home. I have breakfast at work. Their dad gives them breakfast.' (Sabina, 40 years)

In regard to meal composition, parents and adolescents described how breakfast was a meal that had generally become completely 'Australian', with cereal and toast eaten. A Somali mother summed it up:

'Every day, it's normal we have what the Australians have for breakfast. Could be cereal... different kinds of cereals for breakfast and bread.' (Musha, 34 years)

Similarly a Sudanese mother felt that her children had adopted Australian food for breakfast:

'For breakfast we only have tea but kids will have cereal.' (Abra, 37 years)

One Eritrean mother represented the views of many when she explained:

'I don't want them to feel too different... like I want them to mix in with Australians, you know, with the other kids. Because they go somewhere birthday or anything and they see sausage I don't want them to be like "I don't eat this blah blah". I don't want that. You want them to be able to eat everything.' (Makulata, 38 years)

The convenience of Australian food was described by a Sudanese mother:

'It takes long so I just give them Australian food, much easier to cook.' (Fatu, 32 years)

Parents outlined a number of reasons for their children's refusal to eat traditional food. First, because African food 
was considered impractical for children to take to school as it is usually eaten hot and the children did not have access to a microwave. Second, parents believed their children rejected traditional food because it was 'too hot and spicy' for them as growing up in Australia resulted in a preference for mild food. A Sudanese father described the ease of Australian food:

'... [Australian] food doesn't require heating... you

know you can make it quickly...' (Karibu, 39 years)

An Ethiopian mother added:

'Too strong for them [the children]. And hot. Our food is normally spicy.' (Sabina, 40 years)

Parents were receptive to nutrition and health promotion messages in Australian society and commented on how they looked for the 'Heart Foundation Tick' and read food labels, nutrition messages and nutrition information panels presented on food packages when shopping to assist them to make healthier choices. An established Ethiopian father used well-known nutrition symbols such as the Heart Foundation Tick to assist him in choosing 'healthier' products when shopping:

'Of course I look at packages when shop. Every shop look for Heart Foundation Tick. Need to look at every box... [I compare] cornflakes and homebrand ones and home-brand is cheaper and less calories, less fat.' (Musa, 35 years)

An Ethiopian mother described her health-oriented shopping practices:

'Sometimes when I shop yes I look... I have to look which one I buy like cholesterol free. Most of them say that. Like light... Most of the time I check fat.' (Alberta, 38 years)

A Somali mother similarly described how she would read food labels to help her make healthy choices:

'I read... like you know skim what's it called? Not full cream, skinny milky, healthy and all that... our tea we put lots of sugar... so yes we worry about it and my friends if they come and I wanted to make cup of tea and I think maybe I [should] make the sugar less... we have a lot of diabetes [in our community]... We fry food with oil. Here [in Australia] used to boiling food. Healthier cooking here. Plain but doesn't taste nice. Still fry here because we are used to it.' (Maca, 34 years)

\section{Adolescents'views}

Like parents, the adolescents similarly identified two modes of acculturation: lifestyle changes and dietary changes.

\section{Lifestyle changes}

Adolescents felt that the lifestyle in Australia was much easier than in Africa. Technology such as telephones, vacuum cleaners and washing machines had removed the exercise provided by hard, manual labour done back home. Young girls spoke about getting down on their hands and knees and vigorously mopping floors when cleaning the house in Africa. Visiting friends was also a more sedentary process in Australia with adolescents describing how in Australia they could ring friends and organise to meet up, whereas in Africa they would physically walk to their friends house (which could be many kilometres away) to see if they were free. In addition, the adolescents felt that the busier lifestyle had reduced the community feeling that they were familiar with in Africa. The adolescents felt that in Australia people had 'their own agenda', had less time available for others and were always complaining of being busy. A Somali girl described the greater amounts of physical labour conducted in Africa compared with Australia:

'But in Africa it's more like you know it's not like this easy life, it's more like hard working you know, you use your body working... that's why people seem to be more fit but then here [in Australia] there's dish washers, there's washing machines. In Africa you have to get your whole arms [on the floor] to mop...' (Ushab, 16 years)

Another Somali girl supported her views that the Australian lifestyle was less physically demanding:

'You do more walking [in Africa], let's just say your close friend lives somewhere a bit far from here... you could actually walk there you know, its like a normal thing... like there is no telephone, "hey I'm coming over"... you just get your neighbour knocking on your house "hello", "hey what a surprise!"... Not here, everyone's... like "I'm busy, I can't do this and I can't do that", more like there [in Africa] people are helping each other more so it's not like a busy life you know... Whereas here people just have their own transport, like why walk when I can just call her [my friend]...' (Kobia, 15 years)

\section{Dietary acculturation}

In contrast to parents, the adolescents described changes in food habits as part of their dietary acculturation. Dissonance between how frequently traditional food was eaten was evident between parents and adolescents. While parents consistently stated that their children preferred Australian food and ate traditional food infrequently, the adolescents felt that they ate traditional food every day. The dissonance may have arisen from parents' expectations for children to eat traditional food for all meals, while adolescents felt they were eating enough traditional food to satisfy their parents' expectations. Adolescents described opting to eat traditional food especially at special occasions such as weddings or 
birthdays to satisfy their parents but that they would prefer to not eat it on a daily basis. As an Ethiopian boy explained:

'I don't find it disgusting. I just don't want to eat it as much as they [parents] do. If it's like a special occasion like a wedding or something or someone comes to our house then I might eat with them but if it's generally just like for dinner I won't eat it. I'll eat cereal or something like that.' (Nzonio, 14 years)

Although young people contradicted parents' assertion that they rejected traditional food, there was a consensus around a preference for Australian food (Table 2). For young people, Australian food was 'cheap', 'tasty' and 'faster' and eaten by their peers. In contrast, African food was described as 'boring', 'monotonous' and 'time-consuming' to prepare. Like the parents, the young people also described African food as being impractical to take to school as it required heating and was best eaten hot and fresh. One Sudanese girl explained how lack of cooking knowledge hindered the adolescent's ability to prepare traditional food. In addition, the amount of time involved in making traditional food encouraged children to opt for easier and faster foods:

'Kids don't want African food, ate it for a long time. Kids want sandwich, KFC, Maccas like because it is new and easy. Our food takes long time to cook and kids don't know how to cook it. Takes about 2 hours. When only 10 to 12 [years old] can't make it [African food] now they do make a sandwich and other things. Eat it.' (Chiata, 16 years)

One Ethiopian boy explained how he had been encouraged to try burgers after seeing his peers eating them:

'You might want to buy a burger just like friends... when I came from Africa and I never knew any burgers but my friends at school were eating burgers and I thought hey I'm gonna give that a shot and then I tried it and I digged it.' (Solomo, 14 years)

Religious-bound values related to food influenced food choices for Muslim Horn of Africa adolescents. The young Muslims upheld their religious values in relation to eating 'halal' food despite the fact that there was often a limited range of choices available, especially in the school setting. Some Muslim adolescents explained how they could only eat at school one or two days per week when the school canteen would provide halal food. However, the children also spoke about a greater array of halal foods becoming available and that they could now even eat an Australian meat pie and buy 'halal pies' from their local bakeries. A Somali girl explained her inability to eat food provided by the school canteen on certain days:

'On Thursday I bring something else, I can't eat it [school canteen food]. It's not halal.' (Arabia, 15 years)
Unlike the parents, the adolescents did not allude to changes in the timing or composition of family meals post-migration. However, they did feel that they ate fewer meals at home. The adolescents explained that if they did eat at home it would usually be by themselves, due to other family members having already eaten or not being home for meals. Both boys and girls reported erratic eating behaviours and commonly skipped meals most days. Reasons for skipping meals revolved around being late for school, lack of motivation to make a meal and poor appetite. One Sudanese boy represented the views of many of the boys when he explained how his social life impeded his ability to eat with the family:

'I eat alone... Because they [family] have set time to eat and we're never home. We're always at soccer or playing basketball or going out with the boys.' (Koblia, 15 years)

Skipping meals, especially breakfast, was common in both girls and boys. As explained by a Somali girl:

'Well I've realised I eat at school and then I eat at home again... That's weird, two lunches... But I don't eat dinner... I never eat breakfast.' (Buleza, 14 years)

This view was supported by one of the Somali boys:

'I don't eat breakfast here [in Australia]... I get up in the morning and all I want to do is get a shower and go to school. There is not time for you to wait and make food and stuff...' (Kengoti, 15 years)

\section{Theme 2: Food beliefs and perceived bealth consequences Parents' views}

Among parents, food beliefs and perceived health risks were often religious-bound. For Horn of Africa refugees of Islamic faith, religious beliefs appeared to influence self-perceptions about health risk and health status. There was a belief that their life and health were in 'Allah's hands' and they exercised little control over it. While Muslim parents spoke of the role of healthy eating and regular exercise in achieving and maintaining good health, they strongly believed that whether or not they developed a disease was 'God's decision'. The development of a dietary-related disease was generally not considered to be the outcome of other factors such as genetics, diet or lifestyle. Some noted that if they developed diabetes or high blood pressure this was part of 'God's plan' for them. A Somali mother summed up the view of Muslim respondents well when she explained:

'I don't worry if the doctor say you have diabetes or high cholesterol, maybe I listen to what the doctor says to minimise it but not to worry about it. Ok because that's up to God... because of the Muslim 
tradition nobody knows what will happen to them... your life comes from God. No matter how you think you are doing well, everything depends on God, what you get and what you don't get... it doesn't worry myself or my children, we will just cook and eat and we don't care. We trust God to save us.' (Maca, 34 years)

Some non-Muslims identified other factors they thought were risk factors for the development of chronic diseases. These included genetic predisposition, dietary traits especially large portion sizes, the role of individual nutrients (i.e. fat, sugar and salt) and environmental exposure. Particular cultural food habits were considered by parents to represent a significant health risk. For example, traditionally tea is prepared with a large amount of sugar and this was blamed by some parents as the cause of the increase in overweight and obesity observed in the Horn of Africa community. The role of individual nutrients was described by a Sudanese mother:

'Everybody knows if you drink too much sugar you will get sick, if you add too much salt you have sick. In Africa sometimes elderly people have it [diabetes]. For young people not much... But here [in Australia] everybody has diabetes. Eritreans a lot, most of them have diabetes. All my cousins have [diabetes] from my mothers side.' (Mohamudu, 30 years)

A Somali mother described the link between traditional tea and weight gain:

'Have [our traditional] tea with a lot of sugar and milk... so we are getting heavier and heavier.' (Clarice, 34 years)

Possession of certain genes was seen to determine whether a person developed certain diseases or not. The role of age was also implicated in the development of dietaryrelated diseases and established parents reported that obesity and diabetes could affect people at any age. A Sudanese mother explained the role of genetics:

'...if there's any disease it's according with that family, contain that information within the gene family, so you don't see that you get that [diabetes] much outside food or anything...' (Karibu, 39 years)

Dietary-related diseases and lack of physical activity were seen to represent the main health consequences of Australian food and lifestyle. The dietary-related diseases identified included obesity, diabetes and CVD:

'I know how I gained the weight. I don't do a lot of activity with swimming and those stuff. [My daughter]... is 17 and 73 kilo. I worry about her and I always say to her "Don't eat this, don't eat that. Stop that. Go out, play soccer, go to the hip hop". I worry about her.' (Alabchia, Somali father, 38 years)
'Sugar means diabetes. Salt means blood pressure. Fat means cholesterol.' (Abra, Sudanese mother, 37 years)

A Sudanese father demonstrated his concern:

'[We are] worried about the sugar in the blood that is diabetes...' (Karibu, 39 years)

However, many parents were keen for their children to put on weight as this was a sign of prosperity in their homeland and was also thought to increase marriage prospects. A Somali father expressed his desire for his daughter to put on weight:

'I tell her [my daughter] she's too slim and I tell her to eat fatty food. I push her to eat meat.' (Musa, 35 years)

Similarly, a Sudanese mother expressed her support of a higher body weight:

'Normal when fat. That's ok, that's healthy. Our fat is not obese, our fat is healthy, beautiful. You can be big and healthy.' (Zultina, 36 years)

\section{Adolescents' views}

Congruent with the parents' findings, the adolescents identified their perceptions of the health risks associated with consumption of Australian food; the main emphasis being on diabetes. In parallel with the parents' findings, the role of individual nutrients in the development of dietary-related diseases was alluded to by adolescents. Similarly, the adolescents failed to acknowledge a 'whole diet concept' in disease progression nor did adolescents refer to overweight or obesity as a risk factor for other dietary-related diseases such as type 2 diabetes or heart disease. The need to limit certain nutrients to prevent dietary-related diseases was acknowledged by a Somali girl:

'Like for my mum, cause she has diabetes so she tries not to drink or eat anything that has sugar in it... like sweets or stuff like that...' (Wangapi, 14 years)

Interestingly the young people did not feel that they were 'at risk' of developing dietary-related diseases. In contrast, the adolescents described their parents as being significantly 'at risk' for a number of reasons. These included parents' continuation of cultural eating and drinking traditions, lack of physical activity and age. The young people spoke of their parents' continuation of cultural food habits, such as adding significant amounts of sugar ( 4 teaspoons or more) to drinks and cooking with large amounts of oil. The adolescents explained how they rejected these cultural food habits and this helped to protect them from developing dietary-related diseases:

'I think it's because they're [parents] getting old... My aunty was diagnosed a diabetic and I was just 
like "don't worry aunty you're just growing old"... she's about fifty and she has diabetes and she's like "...be careful what you eat, don't eat too much sugar, don't do this, don't do that" and I'm like "oh you're just growing old... relax we're the youngest you know".' (Omba, Somali boy, 15 years)

'Because you burn it [burgers] out during the day, see at lunchtime you have the burger and for the rest of the day you play footy or soccer or rugby... the whole food processes down...' (Kapita, Ethiopian boy, 15 years)

The girls explained how their mothers were always making excuses for not exercising, such as, being too old, tired and not wanting to wear 'sports clothing'. A Somali girl explained:

'[The mums tell me] "I'm putting on weight"... and I'll be like "have you guys tried walking?"... and they'll be like "nah I'm too old"... And they say were just too embarrassed to wear runners... "oh my god I'm not going to wear a skirt with runners, how stupid do you think I look, I'm your mum ok?".' (Arabia, 15 years)

\section{Theme 3: Parental feeding practices}

This theme emerged only from parents' findings and encompassed the role of parenting styles and the use of feeding strategies to regulate children's dietary behaviours. Regulation of children's dietary behaviours was a focal point for parents in order to attain their desired goal of family commensality. Parents displayed a strong desire for children to retain cultural food habits, limit consumption of Australian foods and take part in family, home-cooked meals. Parents felt that their children were deviating away from family commensality and this resulted in deterioration of the identity of the family. Through parental feeding strategies, the parents attempted to achieve and maintain family commensality. Different parenting styles were evident in the parents' transcripts, with two main forms identified: authoritarian-like and authoritative-like. Authoritarian-like parents were predominantly newly arrived refugees. These parents displayed a strong desire to control their children's eating habits. In contrast, authoritative-like parents were more willing to accept input from their children regarding eating habits and gave their children an opportunity to assist in food decision-making processes. Parents who demonstrated authoritative-like parenting characteristics were generally more established and the majority were mothers.

Authoritarian-like or controlling parents conducted a high level of control over their children's diet in regard to what they consumed and where. Parents attempted to control what food their child ate, where they ate it and who they ate it with. Controlling feeding strategies included: (i) controlling eating habits; (ii) restricting food choices; and (iii) using Australian food as a reward. For example, Australian food ('junk food') was used as an incentive to encourage children to eat traditional food. If children ate and finished their traditional food first then they were entitled to a 'reward' of Australian food. In one instance, a mother explained how she had bribed her children with McDonalds to persuade her children to eat a family-cooked meal. In most cases, input from the child regarding food choices was either discouraged or prohibited. These parents encouraged children to eat traditional food at home to achieve family commensality, regulate their social life and keep them at home more often. These children were also not allowed to leave the house on their own and buy 'outside food'. If children bought 'outside food' with friends this was considered to be highly disrespectful and breaking cultural traditions. As explained by a Sudanese mother:

'Kids trying to be responsible for themselves when not supposed to. Is big problem in our culture... Doesn't matter how old they are, [if they are] not married they cannot leave the house and eat elsewhere, must be at home to eat. Not married, don't go out alone.' (Abra, 37 years)

This was supported by a Sudanese father:

'They come home for the time they want to eat then they go out and then they come home again, that way keeps them at home... if you allow your child to eat only the junk food or the restaurant's food, she or he will not be able again to eat the food you cook at home and that encourage the kid to stay outside longer...' (Karibu, 39 years)

A Sudanese mother similarly noted:

'Parents say to children if you don't eat traditional food you can't have Australia food. They have no choice, eat African food or they won't get any.' (Conciata, 40 years)

The use of Australian food as a reward was well summed up by a Somali mother:

'...I always say to them, have the food that I cook first and when you had that you can and if you feel like you want to eat something else then that is maybe when you have, the special one, the Australian food...' (Musha, 34 years)

Parents who appeared to exhibit an authoritative-like parenting style were more lenient on their child's eating habits and gave their child greater input towards their food choices. For example, one mother explained how her children were encouraged to take turns in choosing what they wanted the family to eat on 'Friday takeaway night'. These parents also recognised that as children grew and entered adolescence parents had little to no control over what their child ate (due to a disposable income from 
part-time work for example) and accepted this. A Somali mother described how she gave her children input over their food choices:

'If they want to choose what to have Friday night and the kids want... once a week pizza then they can have takeaway pizza.' (Olga, 39 years)

A Somali recognised the fact that as children grew into adolescents, parents were less able to control their child's dietary intake:

'For me [junk food] once a week is ok. When the children grown like 15-16 they're free to choose what they want. When they're in primary school, we give once or twice a week.' (Makulata, 38 years)

An established Ethiopian father demonstrated how he did not control his daughter's food choices and would simply guide and assist her to make the best decision:

'Food is not compulsory, not a big deal. [My daughter] she choose what she want to eat... I don't think fathers or parents should tell their kids what to do should just give guidance. Be supportive.' (Musa, 35 years)

\section{Discussion}

The present research found that acculturation occurred in the study population in two different forms: changes in lifestyle and dietary acculturation. These findings are congruent with previous studies among migrants from South America, Asia, South-East Asia and Africa to the USA, Canada and Australia ${ }^{(10,13,22-25)}$. The findings from the study suggest that lifestyle changes encountered by refugees related to adapting to the fast-paced and time-constrained lifestyle in Australia which, coupled with a cooler climate, created less time available for physical activity hence increasing the risk of obesity. This is supported by a study by Renzaho et al. ${ }^{(9)}$ which examined lifestyle changes among sub-Saharan African migrants and identified four levels of acculturation: integration (accepting both African and Australian ways of life), assimilation (rejecting African orientation and fully accepting Australian ways of life), marginalisation (rejecting both African and Australian orientations) and traditional (maintenance of Africa orientation and rejection of Australian way of life). Migrants who were more assimilated were found to be more sedentary while maintenance of traditional orientation was associated with better nutritional outcomes. The finding from the current study that migrating to a developed country is associated with a more sedentary lifestyle is consistent with other studies involving African migrants in Melbourne, Australia $^{(26)}$. In addition, the finding that a cooler climate may reduce physical activity levels in migrants is consistent with international studies. A Canadian study found that the cooler climate in Canada led to decreased physical activity levels in South Asian Fijian women post-migration ${ }^{(23)}$.

In the current study, dietary acculturation was characterised by children's preference for Australian foods and rejection of traditional foods, parents' adoption of health-oriented shopping and cooking practices, and fathers taking on a greater role in meal preparation and provision. These findings are consistent with the current literature ${ }^{(10,13,22,24,27-29)}$. An interesting finding from the present study was in relation to the mothers' frustration at their children's willingness to reject traditional foods and abandon family commensality. Mothers disapproved of accommodating children's food preferences for Western convenience foods, such as pizza, hamburgers and spaghetti, and insisted that their children ate the traditional food they had prepared at home as a family. The preparation and provision of meals has been previously identified as central to a mothers' sense of value, moral worth, self-fulfilment and identity ${ }^{(30)}$. The desire of the mothers in the present study to maintain family commensality of traditional meals reflects their desire to demonstrate, maintain and affirm their own identity. Another form of cultural identity connected to food preparation that has been documented in the literature among African migrants to developed countries is that of the serving of meals to husbands. A Swedish study found that Somali women were concerned with the loss of identity attributed to failing to prepare and serve meals to their husbands due to less time available with women entering the workforce ${ }^{(31)}$. However, the need to serve husbands to affirm and maintain cultural identity was not alluded to by the mothers in the current study.

Dietary-related diseases including obesity, diabetes and CVD were identified as the major health consequences associated with dietary changes post-migration and this was consistent across the ethnic groups included in the present study. However, there were a few exceptions, where Muslims tended to believe that the development of these diseases was 'Allah's decision'. Perceived health risks included the role of individual nutrients in disease development (i.e. salt and high blood pressure, sugar and diabetes), lack of physical activity and some specific cultural food habits (i.e. addition of large amounts of sugar to drinks). The role of obesity as a risk factor for dietary-related diseases was not discussed by any of the study participants. While every adolescent could name an immediate or close family member with a chronic dietaryrelated disease, particularly diabetes, they did not perceive themselves to be 'at risk' of developing these diseases. The adolescents claimed that their youth, high levels of physical activity and rejection of the 'problematic' cultural food habits protected them from these diseases. The perceived health risks and consequences associated with dietary changes post-migration have not been previously documented among African migrant groups. In the current study, both parents and adolescents identified individual 
nutrients as risk factors for dietary-related diseases. In contrast, a study among British Bangladeshis identified an imbalance in the consumption of 'strong' and 'weak' foods as a risk factor for diabetes post-migration ${ }^{(32)}$. This suggests that perceived health risks associated with dietary-related diseases vary significantly among migrant groups according to their country of origin and appear to be culturally derived.

In regard to parental feeding practices, parents in the current study displayed a strong desire for children to retain cultural food habits, limit consumption of Western (Australian) foods and maintain family commensality. Regardless of their level of acculturation and the length of stay in Australia, parents feared that a loss of cultural values such as family commensality would coincide with a loss of family closeness and hence parents employed a number of different feeding strategies to coerce their children into maintaining traditional eating habits. Parents would achieve control of their children's eating habits by demanding that children came home to eat and they were prohibited from eating 'outside food' from fast-food restaurants with friends. The goal was the preservation of cultural values related to eating; rejection of cultural values was seen as a significant source of conflicts between parents and children. Renzaho et al. ${ }^{(33)}$ similarly found a greater emphasis on preservation of cultural values and family closeness by Arabic-speaking migrant parents and their children. In an intergenerational study involving African migrant parents and adolescents in Melbourne, Australia, it was found that parents wanted to reinforce pre-migratory beliefs related to food practices whereas children would find coping mechanisms to withstand these parental pressures ${ }^{(34)}$. However, among parents who were integrated and had been in Australia for a long time, they exhibited an authoritative approach to parenting, characterised by bribing children with Australian foods and using it as a 'reward' for children to retain some of their cultural values. One mother described giving her children the reward of a McDonalds 'happy meal' for taking part in and finishing a traditional family meal. Such rewards were facilitated by the exposure to far more food choices both in terms of accessibility and affordability, and such food abundance influenced individual food choices and allowed parents to use food as a tool for negotiating with children, rather than the punitive approach that characterised their pre-migration parenting style ${ }^{(33)}$. In contrast, food was used for restricting and controlling children's decisions and behaviours among newly arrived parents $(<5$ years in Australia) and those who maintained traditional orientation. The use of fast food as a reward for children among African migrants in Australia has been previously identified in the literature ${ }^{(26)}$. The literature is scarce in relation to identifying the consequences of parental feeding strategies employed by migrant parents. In spite of this, existing data in Caucasian populations suggest that feeding strategies such as the ones exhibited by the parents in the present study (i.e. controlling, restricting and rewarding) can affect a child's ability to self-regulate appetite and hence increase the risk of the child becoming overweight or obese ${ }^{(35)}$.

The use of food as a reward has been documented in studies among Australian parents ${ }^{(36)}$. Australian parents were found to frequently use dessert as a reward to encourage their children to finish their meals, in response to parental concern that the child had not eaten enough. The use of food rewards by Australian and African migrant parents differs in that African parents in the current study used rewards to maintain traditional family food practices. However, similarities do exist among Caucasian and migrant parents in the use of food rewards to encourage children to 'finish' meals, which has been documented among both Caucasian ${ }^{(36)}$ and Hispanic migrant parents ${ }^{(37)}$. Studies investigating parental feeding strategies in migrant groups are limited to Hispanic migrants in the USA ${ }^{(37-39)}$. Kaiser et $a{ }^{(39)}$ found that more acculturated Hispanic mothers were less likely to view bribes, threats and punishments as effective feeding strategies. Different methodologies used in these studies make it difficult to obtain a clear picture of the types and purposes of feeding strategies used by migrant parents. Despite previous studies among Hispanic migrants demonstrating that migrant parents certainly employ feeding strategies to attain desired eating behaviours in children, none of the specific strategies used by sub-Saharan African migrants were evident in other studies. There is also a limited description of the role of migrant fathers on parental feeding strategies. Furthermore, a lack of demographic data on study participants in previous studies ${ }^{(38)}$, such as migration status, length of stay, socio-economic status or measures of acculturation levels, makes it difficult to take the life experiences of study participants into account and therefore critically compare study results.

The present study encountered methodological limitations commonly encountered in qualitative cross-cultural research ${ }^{(40)}$. Grandparents were not included in the study sample. Inclusion of grandparents may have provided greater insight from the perspective of an older generation into determinants of post-migration food habits and subsequent perceived health risks. In spite of this, two generations (parents and children) were sufficient to show that there are significant intergenerational differences regarding food-related beliefs, attitudes and values and health risks, leading to family conflict. Men were also under-represented in the study sample. However, men are commonly under-represented in research and more so in migrant studies ${ }^{(24)}$. A gendered interest in the domain of food and health may also have encouraged more women to partake in the study. Another potential limitation of the study is that it was not possible to recruit participants by migration status. Preliminary discussions with African community leaders identified questioning about migration status to be a sensitive topic for participants who entered 
as refugees under the humanitarian scheme. We attempted to address this by recruiting participants by country of origin as an indicator of migration status in order to diversify the study sample. In addition, although we used data saturation to guide our sample size, Eritreans were under-represented. It is possible that our sample contained a larger number of migrants who migrated as refugees and therefore the findings from the study may not transferrable to Horn of Africa migrants who migrated with a higher level of educational attainment under the skilled migration scheme.

\section{Policy implications and conclusion}

Findings from the current research highlight the need for health professionals and organisations servicing African migrant communities to be aware of culturally mediated values and beliefs towards food and poor health literacy among these communities, and to ensure that health promotion programmes put in place to address their needs are informed by an acculturation framework. The current research also ultimately highlights directions for future research among sub-Saharan African migrants in Australia and contributes to the current body of knowledge regarding post-migration dietary changes and chronic disease risk perceptions in this subpopulation. The findings from the present study support the need for further research in a number of areas. First, more research is needed to contextualise dietary acculturation among migrant youth. It was not evident from the results whether rejection of traditional food by the adolescents was in general or in relation to specific food items. Additionally, whether the migrant adolescents' rejection of traditional food was related to general 'rebelling' in adolescence or if it persists into adulthood requires further investigation. The rejection of traditional foods and parents' demand for children to consume traditional foods could be a result of poor health literacy in relation to healthy food choices in both generations. Greater understanding is needed in relation to the impact of migration and acculturation on sub-Saharan Africans' parenting styles. Moreover, the influence of parenting styles on feeding practices and child eating behaviours needs to be explored within an anthropological framework. Importantly, the study highlights the need for future research in migrant groups from an intergenerational perspective. This will ensure that the spectrum of views and beliefs about food and health that exist among and between generations is incorporated into community nutrition and health promotion interventions. Lastly, it is imperative that programmes aimed at addressing chronic disease among African refugees and migrants focus on the family environment, complemented with public health campaigns and support from health professionals to increase health literacy in this subpopulation.

\section{Acknowledgements}

Sources of funding: This study was completed while A.W. was studying at Deakin University. A.R. is supported by an Australian Research Council Future Fellowship. The study was funded by the Deakin University Faculty Research Development Grant. Deakin University had no role in the design, analysis or writing of this article. Conflicts of interest: The authors declare that they have no conflicts of interest relevant to this manuscript. Ethics approval: The study was approved by the Deakin University Human Ethics Committee. Authors' contributions: A.W. and A.R. designed the study and all interview guides used in the study as well as the data analysis. A.W. drafted the article. A.R. made substantial contributions in interpretation of the data, intellectual input during the write-up stage and critically reviewed the manuscript. Both authors have approved its re-submission. Acknowledgements: The authors would like to thank the African Review Panel for mobilising the sub-Saharan African migrant population during data collection and for its input in the development of the interview guide. The authors would also like to thank the overall sub-Saharan African community living in Victoria for endorsing the study and generously taking part in it.

\section{References}

1. United Nations Department of Economic and Social Affairs (2009) Trends in International Migrant Stock: The 2008 Revision. New York: UN Population Division.

2. Martin J (2009) Migration and The Global Economy: Some Stylized Facts. Paris: Organisation for Economic Co-operation and Development.

3. Australian Bureau of Statistics (2012) Reflecting a Nation: Stories from the 2011 Census. Canberra: Commonwealth of Australia.

4. Deloitte Access Economics (2011) The Housing Aspirations of New Settlers to Australia. Canberra: National Housing Supply Council.

5. Cappuccio FP, Kerry SM, Adeyemo A et al. (2008) Body size and blood pressure: an analysis of Africans and the African Diaspora. Epidemiology 19, 38-46.

6. Saleh A, Amanatidis S \& Samman S (2002) The effect of migration on dietary intake, type 2 diabetes and obesity: the Ghanian Health and Nutrition Analysis in Sydney, Australia (GHANAISA). Ecol Food Nutr 41, 255-270.

7. Cohen M, Stern E, Rusecki Y et al. (1988) High prevalence of diabetes in young adult Ethiopian immigrants to Israel. Diabetes 37, 824-828.

8. Rubinstein A, Graf E, Landau E et al. (1991) Prevalence of diabetes mellitus in Ethiopian immigrants. Isr J Med Sci 27, 252-254.

9. Renzaho A, Swinburn B \& Burns C (2008) Maintenance of traditional cultural orientation is associated with lower rates of obesity and sedentary behaviours among African migrant children to developing countries. Int J Obes (Lond) 32, 594-600.

10. Renzaho A (2007) Migrants Getting Fat in Australia: Acculturation, Nutritional Outcomes and Physical Activity among African Migrants to Developed Countries. New York: Nova Science Publishers Inc. 
11. Hervey K, Vargas D, Klesges L et al. (2009) Overweight among refugee children after arrival in the United States. $J$ Health Care Poor Underserved 20, 246-256.

12. Satia-Abouta J (2003) Dietary acculturation: definition, process, assessment and implications. Int J Hum Ecol $\mathbf{4 1}$, $255-270$.

13. Renzaho A \& Burns C (2006) Post-migration food habits of sub-Saharan African migrants in Victoria: a cross-sectional study. Nutr Diet 63, 91-102.

14. Berry JW (1997) Immigration, acculturation, and adaptation. Appl Psychol 46, 5-68.

15. Flannery WP, Reise SP \& Yu J (2001) An empirical comparison of acculturation models. Pers Soc Psychol Bull 27, 1035-1045.

16. Padilla AM \& Perez W (2003) Acculturation, social identity, and social cognition: a new perspective. Hisp J Behav Sci 25, 35-55.

17. Renzaho AMN (2009) Immigration and social exclusion: examining health inequalities of immigrants through acculturation lenses. In Theorising Social Exclusion, pp. 117-126 [A Taket, B Crisp, A Nevill et al., editors]. Oxford: Routledge.

18. Wilson A, Renzaho A, McCabe M et al. (2010) Towards understanding the new food environment for refugges from the Horn of Africa in Australia. Health Place 15, 969-976.

19. Parker R \& Kindig D (2006) Beyond the Institute of Medicine health literacry report: are the recommendations being taken seriously? J Gen Intern Med 21, 891-892.

20. Minichiello V, Madison J, Hays T et al. (1999) Qualitative interviews. In Handbook for Research Methods in Health Sciences, pp. 395-418 [V Minichiello, G Sullivan, K Greenwood et al., editors]. Sydney: Addison-Wesley.

21. Ezzy D (2002) Qualitative Analysis: Practice and Innovation. New York: Routledge.

22. Bermúdez OI, Falcón LM \& Tucker KL (2000) Intake and food sources of macronutrients among older hispanic adults: association with ethnicity, acculturation, and length of residence in the United States. J Am Diet Assoc 100, 665-673.

23. Elliott SJ \& Gillie J (1998) Moving experiences: a qualitative analysis of health and migration. Health Place 4, 327-339.

24. Ristovski-Slijepcevic S, Chapman G \& Beagan B (2008) Engaging with healthy eating discourse(s); ways of knowing about food and health in three ethnocultural groups in Canada. Appetite 50, 167-178.

25. Satia J, Patterson R, Neuhouser M et al. (2002) Dietary acculturation: applications to nutrition research and dietetics. J Am Diet Assoc 102, 1105-1118.

26. Renzaho A, Green J, Mellor D et al. (2011) Parenting, family functioning and lifestyle in a new culture: the case of
African migrants in Melbourne, Victoria, Australia. Child Fam Soc Work 16, 228-240.

27. Burns C (2004) Effect of migration on food habits of Somali women living as refugees in Australia. Ecol Food Nutr $\mathbf{4 3}$, 213-229.

28. Devine CM, Sobal J, Bisogni CA et al. (1999) Food choices in three ethnic groups: interactions of ideas, identities, and roles. J Nutr Educ 31, 86-110.

29. Green J, Waters E, Haikerwal A et al. (2003) Social, cultural and environmental influences on child activity and eating in Australian migrant communities. Child Care Health Dev 29, 441-448.

30. Counihan C \& Kaplan SL (2004) Food and Gender: Identity and Power. Amsterdam: Harwood Academic Publishers.

31. Jonsson IM, Hallberg LRM \& Gustafsson IB (2002) Cultural foodways in Sweden: repeated focus group interviews with Somalian women. Int J Consum Stud 26, 328-339.

32. Chowdhury AMM, Helman C \& Greenhalgh T (2000) Food beliefs and practices among British Bangladeshis with diabetes: implications for health education. Anthropol Med 7, 209-226.

33. Renzaho A, McCabe M \& Sainsbury W (2011) Parenting, role reversals and the preservation of cultural values among Arabic speaking migrant families in Melbourne, Australia. Int J Intercultural Relations 35, 416-424.

34. Renzaho A, McCabe M \& Swinburn B (2011) Intergenerational differences in food, physical activity and body size perceptions among African migrants. Qual Health Res 22, 740-754.

35. Faith MS, Scanlon KS, Birch LL et al. (2004) Parent-child feeding strategies and their relationships to child eating and weight status. Obes Res 12, 1711-1722.

36. Campbell KJ, Crawford DA \& Hesketh KD (2007) Australian parents' views on their 5-6-year-old children's food choices. Health Promot Int 22, 11-18.

37. Sherry B, McDivitt J, Birch LL et al. (2004) Attitudes, practices, and concerns about child feeding and child weight status among socioeconomically diverse white, Hispanic, and African-American mothers. J Am Diet Assoc 104, 215-221.

38. Gomel JN \& Zamora A (2007) English- and Spanishspeaking Latina mothers' beliefs about food, health, and mothering. J Immigr Minor Health 9, 359-367.

39. Kaiser L, Melgar-Quinonez HR, Lamp CL et al. (2001) Acculturation of Mexican-American mothers influences child feeding strategies. J Am Diet Assoc 101, 525-547.

40. Williamson A \& De Souza R (2007) Research with Communities: Grounded Perspectives for Engaging Communities in Research. Auckland: Muddy Creek Press. 